\title{
Clostridium tertium Empyema after a Hemi-Colectomy and Repair of Incarcerated Hernia
}

\author{
Fahad Alroumi, ${ }^{1,3}$ Joshua Giaccotto, ${ }^{1,3}$ Akmal Sarwar, ${ }^{1,3}$ and Daniel P. McQuillen ${ }^{2,3}$
}

\begin{abstract}
Background: Clostridium tertium is a spore-forming, anaerobic gram-positive bacilli. It is found in soil as well as in human oral and intestinal flora. Unlike other clostridia, Clostridium tertium is aerotolerant, non-toxinproducing, and has been described only rarely as a human pathogen. Most case series involve bacteremia possibly originating from mucosal translocation. Other reports have described skin, soft tissue, bone, and central nervous system infection. Its involvement in the lung has been limited to only a few cases.

Case Presentation: We report a case of empyema caused by Clostridium tertium that occurred shortly after a hemi-colectomy and repair of an incarcerated umbilical hernia. We describe its successful treatment with chest tube drainage and antimicrobial therapy.
\end{abstract}

Keywords: clostridium; empyema; infection; pleural effusion; pneumonia

T HE GENUS Clostridium exist as intestinal flora and in some cases are pathogenic [1-3]. Clostridium tertium is aerotolerant, permitting growth in an aerobic environment [1-3]. In contrast to other species of the same genus, this organism does not produce toxins [1]. It was first isolated in 1917 from war wounds but it was not until 1963 that the organism was first recognized as a human pathogen after a case of $C$. tertium septicemia was described by King et al. [5].

\section{Case Presentation}

A 75-year-old male with a history significant for obstructive sleep apnea, obesity, diabetes mellitus, asthma, and chronic urticaria presented with a three-day history of worsening dyspnea, intermittent pleuritic chest pain, and anorexia without cough or fevers. He had undergone a repair of an incarcerated umbilical hernia and a right hemi-colectomy nine days prior. Intra-operatively, a strangulated portion of bowel was noted within the hernia. There was necrosis of the bowel as it approached the ileocecal junction. He had received cefazolin, ciprofloxacin, and metronidazole pre-operatively and had only been discharged three days before this presentation. He was taking prednisone $10 \mathrm{mg}$ daily as long-term steroid therapy for chronic urticaria and budesonide-formoterol with albuterol for asthma. The patient was a lifelong non-smoker.

On examination, vital signs were within normal limits. Lung auscultation disclosed diminished right-sided breath sounds. There was a mid-line laparotomy scar on the abdomen that appeared clean, dry, and intact. Serous material exited the patient's right flank via a Jackson-Pratt drain.

Laboratory investigations on admission revealed an elevated white cell count $\left(16.65 \times 10^{9}\right.$ cells per liter $)$ with a neutrophil predominance $(86 \%)$. Renal, hepatic, and coagulation parameters were normal. Beta natriuretic peptide was within normal limits but albumin was low at $1.9 \mathrm{~g} / \mathrm{dL}$. On chest radiography, a moderate right pleural effusion was present (Fig. 1). A computed tomography (CT) angiogram of the chest demonstrated a loculated right pleural effusion with compressive atelectasis of the right middle and lower lobes (Fig. 2).

Ultrasound-guided thoracentesis yielded pleural fluid protein to serum protein ratio of 0.75 . Pleural fluid lactate dehydrogenase was elevated at 757 IU/L but $\mathrm{pH}$ and glucose were normal. Cell count of the pleural exudate was 4,377 cells per liter ( $81 \%$ neutrophils). The patient was started empirically on a combination of cefepime and vancomycin intravenously. The anaerobic pleural fluid cultures grew

\footnotetext{
${ }^{1}$ Department of Pulmonary and Critical Care Medicine, ${ }^{2}$ Department of Infectious Diseases, Lahey Hospital and Medical Center, Burlington, Massachusetts.

${ }^{3}$ Tufts University School of Medicine, Boston, Massachusetts.

(C) Fahad Alroumi et al. 2016; Published by Mary Ann Liebert, Inc. This Open Access article is distributed under the terms of the Creative Commons License (http://creativecommons.org/licenses/by/4.0), which permits unrestricted use, distribution, and reproduction in any medium, provided the original work is properly credited.
} 


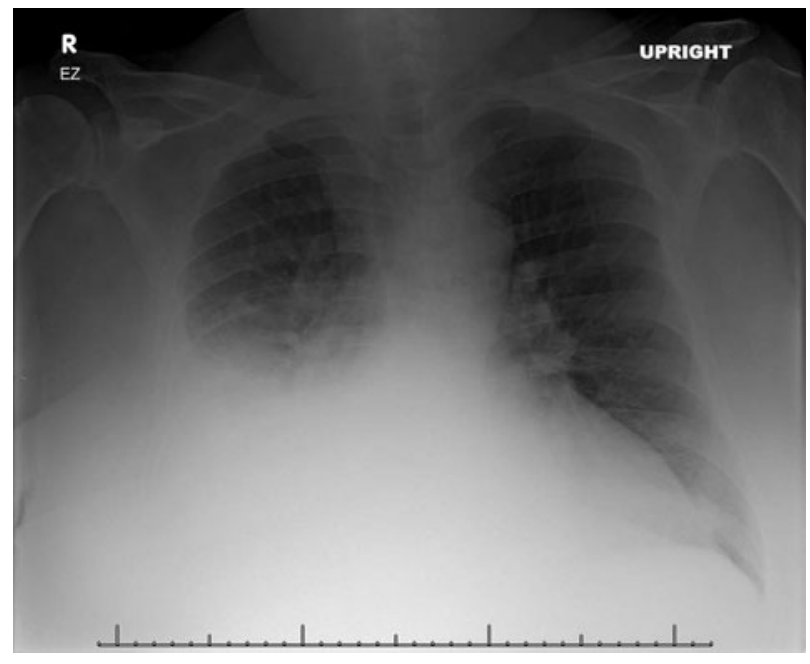

FIG. 1. Posterior anterior chest radiograph showing a moderate right pleural effusion.

aerotolerant gram-positive bacilli after 24 hours. Clostridium tertium was later identified using matrix-assisted laser desorption/ionization time-of-flight mass spectrometry (Bruker Daltonics, Billerica, MA). Methicillin-resistant Staphylococcus aureus was isolated in the sputum culture. Because antimicrobial susceptibility was still pending, the patient was switched to empiric clindamycin and vancomycin (Table 1).

On day two of hospitalization, chest ultrasonography revealed residual loculated effusion prompting CT-guided chest tube placement (Fig. 3). Empyema fluid was cultured and again grew $C$. tertium. Alteplase and dornase alfa were instilled through the chest tube. This was repeated for a total of three doses with notable radiographic and clinical improvement. Because of significant chest pain, the intra-pleural medications were discontinued after three doses. Re-imaging with noncontrast CT chest confirmed a marked decrease in pleural fluid volume, with improved aeration in the consolidated lung.

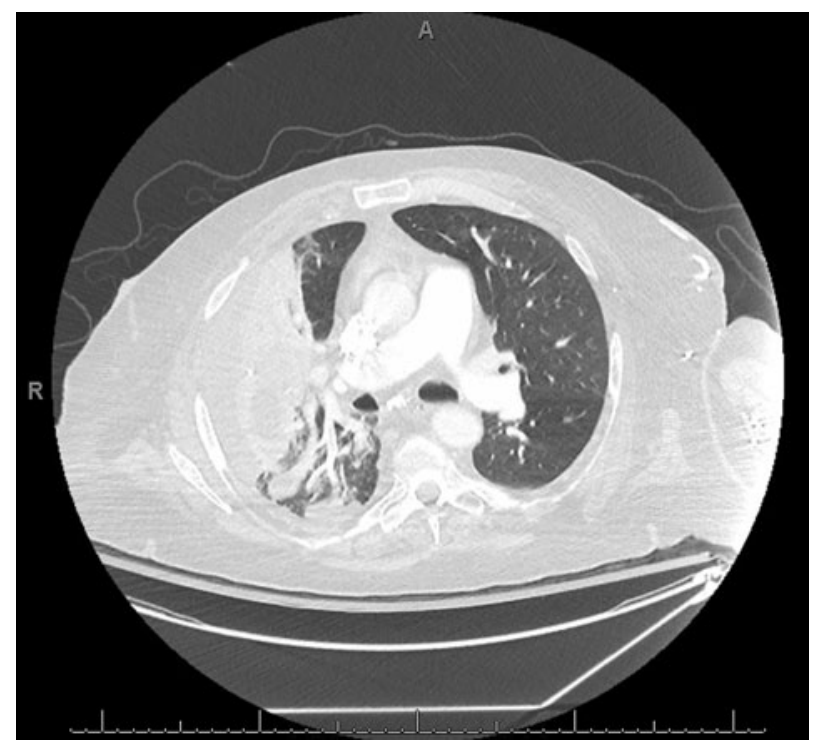

FIG. 2. Computed tomography (CT) of the chest with contrast. There is a moderate loculated right pleural effusion with compressive atelectasis and consolidation in the right upper and lower lobes.
Table 1. Antibiotic Susceptibilities for the Isolated CLOSTRIDIUM TERTIUM

\begin{tabular}{lc} 
Antibiotic & $\begin{array}{c}\text { Minimum inhibit } \\
\text { concentration }(m\end{array}$ \\
\hline Ampicillin/sulbactam & $0.5 / 0.25$ \\
Clindamycin & $\geq 32$ \\
Meropenem & 0.25 \\
Metronidazole & 4 \\
Penicillin & 2 \\
Piperacillin-tazobactam & $8 / 4$
\end{tabular}

There was a small right pneumothorax (Fig. 4). The chest tube was removed after radiographic confirmation of resolution of the pneumothorax. On the basis of susceptibility data available later (Table 1), the patient was discharged on trimethoprimsulfamethoxazole and metronidazole orally to complete a 14day course of antibiotics to cover for both methicillin-resistant $S$. aureus pneumonia and C. tertium empyema.

\section{Discussion}

Despite its low virulence, $C$. tertium has been described in both neutropenic and non-neutropenic individuals $[1-4,6]$. The majority of case reviews identified $C$. tertium as causing abdominal infections or bacteremia believed to have originated from intestinal translocation [1-4,7]. Other case reports have described infections in the form of cerebritis, brain abscess, peri-renal abscess, spontaneous myonecrosis, necrotizing fasciitis, cellulitis, and osteomyelitis. Although there has been a handful of cases of pneumonia with $C$. tertium, there is only one other reported case of empyema described in the medical literature $[4,5,8]$.

Clostridium tertium bacteremia in patients with hematologic malignancy has been well described [1]. The majority of reported cases have described the pathogen in patients who are immunocompromised in some capacity. The major risk factors

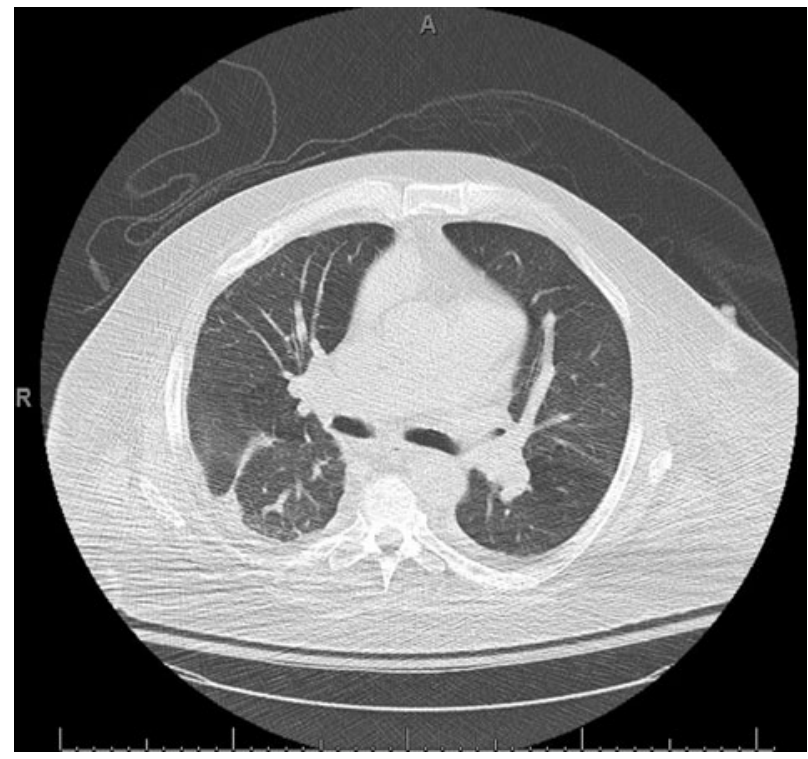

FIG. 3. Computed tomography (CT) of the chest without contrast at the level of the main carina. There is nearcomplete resolution of the effusion and atelectasis at the same level as Figure 2. 


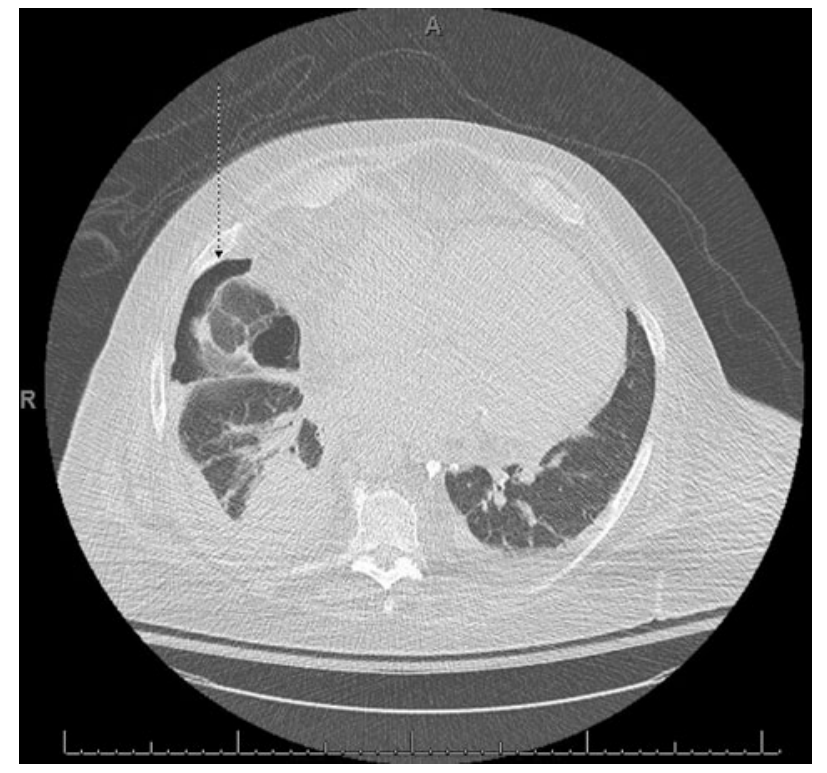

FIG. 4. Computed tomography (CT) of the chest without contrast. There is a small right sided pneumothorax (arrow) from possible air entrainment through the chest tube. There is consolidation of the right lower lobe and a small residual right pleural effusion. There is a small left pleural effusion.

for $C$. tertium infection are neutropenia, intestinal mucosal injury, severe liver disease, and exposure to $\beta$-lactam antibiotics $[1,8]$. Although our patient was not neutropenic, longterm prednisone therapy and a history of poorly controlled diabetes mellitus potentially could have impaired his immune system. He had undergone recent abdominal surgery and had been given cefazolin pre-operatively, which may have put him at risk of selection effect of antibiotics for $C$. tertium.

The aerotolerant characteristics of $C$. tertium enable growth of the organism in an aerobic medium [9]. In these conditions, the bacteria become difficult to differentiate from commensal flora such as Bacillus sp. and Corynebacterium sp. because of shared biochemical traits [8-12]. This presents a diagnostic challenge and possibly contributes to the sparse reports of its pathogenicity. Additionally, some report the bacterium as a contaminant [2]. In our case, the organism was isolated from pleural fluid by thoracentesis initially and a day after when a chest tube was placed. This substantiates its likely role as a pathogen and not a contaminant.

The organism is often susceptible to quinolones, imipenem, vancomycin, and trimethoprim-sulfamethoxazole [9,13]. It is resistant to cephalosporins and clindamycin but susceptibilities to metronidazole vary [4]. In our case, the organism was susceptible to metronidazole (Table 1).

In conclusion, we report a case of empyema caused by $C$. tertium. The patient in our case was immunosuppressed because of long-term steroid therapy and had had recent abdominal surgery. We suspect hematogenous spread of the bacteria to the pleura either peri- or post-operatively. Although its role as a pathogen remains a point of contention, it is clear in our case that it resulted in disease. We report this case to explore further the scope of illness caused by this uncommon organism.

\section{Author Disclosure Statement}

The authors declare no conflicts of interest.

\section{References}

1. Miller DL, Brazer S, Murdoch D, et al. Significance of Clostridium tertium bacteremia in neutropenic and nonneutropenic patients: Review of 32 cases. Clin Infect Dis 2001;32:975-978.

2. Vanderhofstadt M, André M, Lonchay C, et al. Clostridium tertium bacteremia: Contamination or true pathogen? A report of two cases and a review of the literature. Int $\mathbf{J}$ Infect Dis 2010;14(Suppl 3):e335-337.

3. Speirs G, Warren RE, Ramp A. Clostridium tertium septicemia in patients with neutropenia. J Infect Dis 1988;158: 1336-1340.

4. Thaler M, Gill V, Pizzo PA. Emergence of Clostridium tertium as a pathogen in neutropenic patients. Am J Med 1986;81:596-600.

5. King BM, Ranck BA, Daugherty FD, Rau CA. Clostridium tertium septicemia. N Engl J Med 1963;269:467-469.

6. Lee C-S. Clostridium tertium bacteremia in a patient with glyphosate ingestion. Am J Case Rep 2015;16:4-7.

7. Coleman N, Speirs G, Khan J, et al. Neutropenic enterocolitis associated with Clostridium tertium. J Clin Pathol 1993;46:180-183.

8. Johnson JR, Tenover FC. Clostridium tertium bacteremia in a patient with aspiration pneumonia: An elusive diagnosis. J Infect Dis 1988;157:854-855.

9. Henry $\mathrm{CH}$. An investigation of the cultural reactions of certain anaerobes found in wounds. J Pathol Bacteriol 1918; 21:344-385.

10. Fujitani S, Liu CX, Finegold SM, et al. Clostridium tertium isolated from gas gangrene wound; misidentified as Lactobacillus spp initially due to aerotolerant feature. Anaerobe 2007;13:161-165.

11. Ray P, Das A, Singh K, et al. Clostridium tertium in necrotizing fasciitis and gangrene. Emerg Infect Dis 2003;9: 1347-1349.

12. Lew JF, Wiedermann BL, Sneed J, et al. Aerotolerant Clostridium tertium brain abscess following a lawn dart injury. J Clin Microbiol 1990;28:2127-2129.

13. Steyaert S, Peleman R, Vaneechoutte M, et al. Septicemia in neutropenic patients infected with Clostridium tertium resistant to cefepime and other expanded-spectrum cephalosporins. J Clin Microbiol 1999;37:3778-3779.

Address correspondence to:

Dr. Fahad Alroumi

Department of Pulmonary and Critical Care Medicine Lahey Hospital and Medical Center 41 Mall Road Burlington, MA 01805

E-mail: fahad.alroumi@lahey.org

\begin{tabular}{|c|}
\hline $\begin{array}{c}\text { Abbreviation Used } \\
\mathrm{CT}=\text { computed tomography }\end{array}$ \\
\hline
\end{tabular}

Cite this article as: Alroumi F, Giaccotto J, Sarwar A, McQuillen DP (2016) Clostridium tertium empyema after a hemi-colectomy and repair of incarcerated hernia. Surgical Infections Case Reports 1:1, 146-148, DOI: 10.1089/crsi.2016.0041 